Mini Review

\title{
Importance of treating compensatory hypertrophy of inferior turbinate in cases of septal deviation causing nasal obstruction
}

Volume 4 Issue 3 - 2016

\section{Introduction}

Deviated Nasal Septum is one of the commonest disease encountered in Otolaryngology practice where the nasal septum is deviated to one or the other side, one condition that we encounter this condition is increase in the size of Inferior turbinate also called as compensatory hypertrophy of the inferior turbinate is seen in the nasal cavity on the opposite side of the deviation of the septum i.e. if the septum is deviated to right side it will be seen on the left side, this is a natural defense mechanism of the body to prevent excessive use of only one side of the nasal cavity for breathing, and to protect the airways from the excess of air that could enter through the nostril and its potential negative effects such as dryness, alteration of air filtration, mucociliary flow or lung involvement. ${ }^{1}$ The exact cause of this hypertrophy is not entirely clear ${ }^{2}$ but this phenomenon adds to the already existing nasal obstruction and can result in oral breathing and recurrent sinusitis due to impairment of sinus drainage.

\section{Method}

150 patients of Deviated nasal septum with compensatory hypertrophy of inferior turbinate on the contra lateral side were taken in the study. Compensatory hypertrophy was established by Endoscopic methods as well as radiologically (X-ray and CT scans were done) Since no definite criteria of nasal obstruction is available in literature hence a criteria of $4 \mathrm{~mm}$ nasal patency ${ }^{3}$ was taken as adequate and less than that was considered as nasal obstruction (associated with symptoms) Patients were evaluated for a period of one year postoperatively for the regression of compensatory hypertrophy and mostly endoscopy

\author{
Sumit Sharma \\ Department of E.N.T., Mayo Institute of Medical Sciences, India
}

Correspondence: Sumit Sharma, Department of E.N.T., Mayo Institute of Medical Sciences, Barabanki, India, Tel 983-917-8240, Email entsumit@rediffmail.com

Received: May 25, 2016 | Published: June 22, 2016

\section{Results}

Table I Results of the two group after one year of evaluation

\begin{tabular}{lllllll}
\hline & Immediate Post-Operative & 6 Months Postoperative & \multicolumn{2}{c}{ I Year Postoperative } \\
\hline Nasal Obstruction & Group-A & Group-B & Group-A & Group-B & Group-A & Group-B \\
& $75 / 75$ & $50 / 75$ & $75 / 75$ & $50 / 75$ & $75 / 75$ & $45 / 75$ \\
\hline
\end{tabular}

\section{Discussion}

Turbinates are bony projections from the lateral wall of the nose, which are three in number called as Superior, Middle and Inferior turbinate, they provide increased surface area to the nasal cavity and also provide turbulence to the air entering the nasal cavity for sinus ventilation. There is sometimes a fourth turbinate present above the superior turbinate which is called as supreme turbinate. The inferior turbinate is the largest of the three and are responsible for majority of nasal functions. At inspiration up to two-thirds of upper airway resistance is produced by the anterior tip of the inferior nasal concha in the region of the internal nasal valve ${ }^{2}$ and compensatory hypertrophy is most commonly seen in inferior turbinate. It is seen that the inferior was done for evaluation of postoperative results during that period. Patients were put in two groups: GROUP-A=Treated Compensatory hypertrophy $(\mathrm{n}=75)$ and GROUP-B=Compensatory hypertrophy not treated $(\mathrm{n}=75)$.

Surgery for Deviated septum was performed under general anesthesia and compensatory hypertrophy was treated by submucous diathermy (either by electrocautery or Diode laser). Mucosa preservation methods were used and turbinectomy was not done in any case. No significant postoperative complications like bleeding / crusting were encountered. 
In the past it was believed that in compensatory hypertrophy only the mucosal element is enlarged but a histological study by Berger et al. 2000 showed that the most commonly enlarged component of the contra lateral inferior nasal concha in septal deviation is the bony nasal concha.

It has been a topic of debate whether to deal with compensatory hypertrophy of the inferior turbinate at the time of septal surgery or leave it for spontaneous regression which may follow the septal surgery. Studies have shown that spontaneous regression is not commonly encountered as in compensatory hypertrophy the bony component of the inferior turbinate is also involved which does nor regress postoperatively. The surgeries usually performed for this condition fall in two categories, one dealing with the mucosa and the sub mucosa, of inferior turbinate and the other dealing with the bone (Turbinoplasty).

In the present study the post operative results of the patients of septal deviation with compensatory hypertrophy were very different when the compensatory hypertrophy was treated with sub mucous diathermy as compared to those in which it was not treated and left in expectation of decompensation. The initial response of patients in nasal obstruction was much superior (about 100\%) in the treated group as compared to only $50 \%$ relief in untreated group. The percentage did not improve even after 6months and 1year and the proportion remained the same in the two groups suggesting that the changes in the inferior turbinate in compensatory hypertrophy are irreversible and the condition should be treated at the time of correction of the septum to achieve good results in correction of the symptom of nasal obstruction. It is also to be noted that turbinectomy can be avoided to reduce the size of the turbinate and a better method of submucous diathermy (either electrocautry or Lasers) can be used to achieve adequate results, this is in contradiction with the study conducted by Erol Egeli MD et al., ${ }^{5}$ which states that the turbinate bone should be excised to achieve good results in nasal obstruction. The study also contradicts the opinion of Farhad Jalil Khayat ${ }^{6}$ which states that Inferior turbinate hypertrophy, especially in the medial mucosa, reverses 4-6months after septoplasty without turbinate surgery.

\section{Conclusion}

1. It is a well know fact that the inferior turbinate on the opposite side of Deviated Nasal Septum undergoes Compensatory Hypertrophy as a protective mechanism.

2. This Compensatory Hypertrophy can easily be established by Endoscopy and radiological methods (X-ray/ CT scan) taking the contralateral turbinate as reference.
3. It is important to note that this Compensatory Hypertrophy of inferior turbinate involves both the mucosa (predominantly medial turbinate mucosa) as well as the turbinate bone.

4. Treating this Compensatory Hypertrophy at the time of septal correction is advisable as this Compensatory Hypertrophy is irreversible.

5. The best method in the present study was the mucosa preserving submucous diathermy (either using the Electrocautery or the Lasers), as it avoids the major complications of turbinectomy (like bleeding / loss of mucosa / roomy postoperative nasal cavity causing excessive crusting) and gives good results.

\section{Acknowledgments}

None.

\section{Conflicts of interest}

Author declares there are no conflicts of interest.

\section{Funding}

None.

\section{References}

1. Chiesa Estomba C, Rivera Schmitz T, Ossa Echeverri CC, et al. Compensatory hypertrophy of the contralateral inferior turbinate in patients with unilateral nasal septal deviation A computed tomography study. Otolaryngol Pol. 2015;69(2):14-20.

2. Mrig S, Agarwal AK, Passey JC. Preoperative Computed Tomographic Evaluation of Inferior Nasal Concha Hypertrophy and its Role in Deciding Surgical Treatment Modality in Patients with Deviated Nasal Septum. Int J Morphol. 2009;27(2):503-506.

3. Grymer LF, Illum P, Hilberga O. Septoplasty and compensatory inferior turbinate hypertrophy: a randomized study evaluated by acoustic rhinometry. J Laryngol Otol. 1993;107(5):413-417.

4. Berger G, Hammel I, Berger R, et al. Histopathology of the Inferior Turbinate With Compensatory Hypertrophy in Patients with Deviated Nasal Septum. Laryngoscope. 2000;110(12):2100-2105.

5. Egeli E, Demirci L, Yazýcý B, et al. Evaluation of the Inferior Turbinate in Patients with Deviated Nasal Septum by Using Computed Tomography. Laryngoscope. 2004;114(1):113-117.

6. Jalil KF, Mazhar H. Effect of Septoplasty on Inferior Turbinate Hypertrophy. 2012;2(1):21-29. 